\title{
Leishmaniasis mucosa nasolaringotraqueal en un adolescente
}

Nasolaryngotracheal mucosal leishmaniasis in an adolescent

\author{
Dr. Mario Forero ${ }^{a}$, Dra. Alexandra Pérez ${ }^{a}$, Dra. Norma C. Barajas ${ }^{a}$, Dra. Yenny A. Leyva ${ }^{a}$ y Dr. Carlos Zamora ${ }^{b}$
}

\begin{abstract}
RESUMEN
La leishmaniasis es un problema creciente de salud pública, que produce un gran impacto social. La leishmaniasis laríngea y sin puerta de entrada evidente es rara, en especial en pacientes inmunocompetentes. Describimos un caso de leishmaniasis laríngea con extensión traqueal y estenosis secundaria en un adolescente previamente sano y sin factores de riesgo ambiental o personal.

Palabras clave: leishmaniasis, mucosa, laringe.
\end{abstract}

\begin{abstract}
Leishmaniasis is a growing public health problem and it produces a great social impact. Laryngeal leishmaniasis without obvious gateway is rare, especially in immunocompetent patients. We report a case of laryngeal leishmaniasis with tracheal involvement and stenosis on a previously healthy teenager without environmental or personal risk factors.

Key words: leishmaniasis, mucosa, larynx.
\end{abstract}

http:/ /dx.doi.org/10.5546/aap.2014.e101

\section{INTRODUCCIÓN}

La leishmaniasis es una antropozoonosis causada por un protozoario flagelado del género Leishmania inoculado por la picadura de un díptero del género Lutzomia, cuyo principal reservorio en la naturaleza es el perro. ${ }^{1,2}$ Los amastigotes se multiplican en los macrófagos y disparan una respuesta inflamatoria específica compleja, que da lugar a las diversas manifestaciones de la enfermedad. ${ }^{3,4}$ Se describen tres formas clínicas de leishmaniasis: la cutánea, la mucocutánea y la visceral. ${ }^{4}$ Las manifestaciones clínicas generales incluyen fiebre intermitente, cefalea, pancitopenia, hepatoesplenomegalia y emaciación. ${ }^{4}$

La afectación aislada de la mucosa de la vía aérea es infrecuente ${ }^{1} \mathrm{y}$ se asocia principalmente

a. Fundación Cardiovascular de Colombia.

b. FOSCAL.

Floridablanca, Santander, Colombia.

Correspondencia:

Dr. Mario Forero, mforerom@gmail.com

Conflicto de intereses: ninguno que declarar.

Recibido: 22-10-2013

Aceptado: 12-2-2014 con Leishmania braziliensis en Sudamérica. ${ }^{5,6}$ Se han documentado casos secundarios a la reactivación de la forma visceral por Leishmania donovani e infantum. ${ }^{7}$ Hasta el 3\% de las formas cutáneas por Leishmania braziliensis afectan las mucosas concomitantemente o después de 2 años de la remisión, como resultado de la diseminación linfohematógena del parásito. ${ }^{4,6,8}$ El 90\% de las leishmaniasis mucosas afectan la nariz, con menor frecuencia la faringe y es rara en la laringe. ${ }^{4,6,9} \mathrm{La}$ biopsia es necesaria para el diagnóstico en todos los casos, ya que las pruebas serológicas para la detección de anticuerpos tienen una sensibilidad limitada. ${ }^{10}$

\section{CASO CLÍNICO}

Varón de 12 años sin antecedentes de importancia, habitante en zona urbana; presenta episodios de disnea paroxística y fiebre recurrente de 18 meses de evolución. Ingresa a una unidad de cuidado intensivo con diagnóstico de neumonía complicada; presenta múltiples extubaciones fallidas y convulsión tónico-clónica generalizada por hipoxia. Con diagnóstico de estenosis subglótica, es remitido a un hospital de tercer nivel, donde le realizan 3 dilataciones y traqueotomía, y queda con cánula metálica de calibre 3,5 por la gravedad de la estenosis. En la tomografía computada de tórax, se observan calcificaciones ganglionares subcarinales que comprometen la luz de los bronquios y estenosis completa de la tráquea en un trayecto de $11 \mathrm{~mm}$, que incluye la glotis. Por compromiso extenso de la lesión, se difiere la reconstrucción traqueal.

En el seguimiento, se observa aumento del tamaño nasal y masa a nivel septal, cuya biopsia informa tumor mesenquimal de bajo grado. Sin etiología clara, es remitido a nuestra institución. El paciente presentaba lesiones granulomatosas rosadas en fosas nasales (Figura 1); la auscultación cardiopulmonar y la saturación eran normales. El laboratorio mostró hemograma con 13780 leucocitos; neutrófilos, $76 \%$; linfocitos, $20 \%$; $\mathrm{Hb}$, $13 \mathrm{mg} / \mathrm{dl}$; Hto, 38\%; plaquetas, 378 000/ $\mathrm{mm}^{3}$. La baciloscopía seriada, la prueba de Mantoux y los estudios serológicos (VIH, hepatitis B y C) fueron 
negativos. Se efectúa tomografía computada de laringe, en la que se observa lesión de aspecto sólido de crecimiento expansivo, de bordes regulares y bien definidos, que compromete hipofaringe, laringe y tercio proximal de la tráquea y se extiende $3,7 \mathrm{~cm}$ longitudinalmente, $3,4 \mathrm{~cm}$ de diámetro transverso y $2,5 \mathrm{~cm}$ de diámetro anteroposterior, que deja una pequeña luz suboclusiva a nivel distal y con oclusión completa a nivel proximal. En el tórax, se aprecia masa sólida de densidad de tejidos blandos de contornos regulares, que compromete la carina, nivel precarinal e infracarinal de 2,1 por $2,2 \mathrm{~cm}$ con ligera estenosis de los bronquios fuente, predominantemente el derecho, donde la luz se reduce alrededor de un $50 \%$; no se aprecian adenopatías (Figuras 2 y 3 ).

En una junta médica quirúrgica, se decide biopsiar el cornete derecho y la laringe. Para este fin, se realiza nasofibrolaringoscopía bajo anestesia general. Se evidencia epiglotis distorsionada, aumentada de tamaño, con epitelio nacarado vascularizado, vallécula ocupada de forma difusa por tejido blando no friable no ulcerado, cuerdas vocales, repliegues aritenoepiglóticos, cartílago cuneiforme y cartílago corniculado edematizados. Se observa estenosis concéntrica subglótica fija, que impide el paso del broncoscopio flexible a la tráquea a más de $2 \mathrm{~cm}$ de la glotis. Distal a la traqueotomía, la vía aérea era permeable y la mucosa, normal. La muestra de biopsia nasal presentaba estroma fibroconectivo edematoso levemente vascularizado, con escaso aflujo inflamatorio disperso perivascular y pequeños focos de hemorragia sin granulomas ni microorganismos.

FIGURA 1. Lesión granulomatosa rosada en fosa nasal derecha

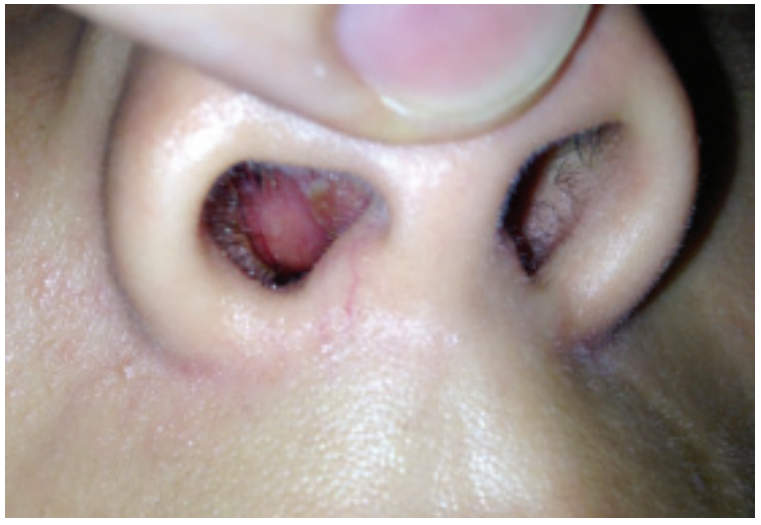

FIGURA 2. Tomografía computada de laringe y tórax, corte coronal. Se observa lesión concéntrica que compromete hipofaringe, laringe y tercio proximal de la tráquea, estrechamiento a nivel precarinal, carinal e infracarinal y estenosis de bronquios fuente de predominio derecho (flechas)

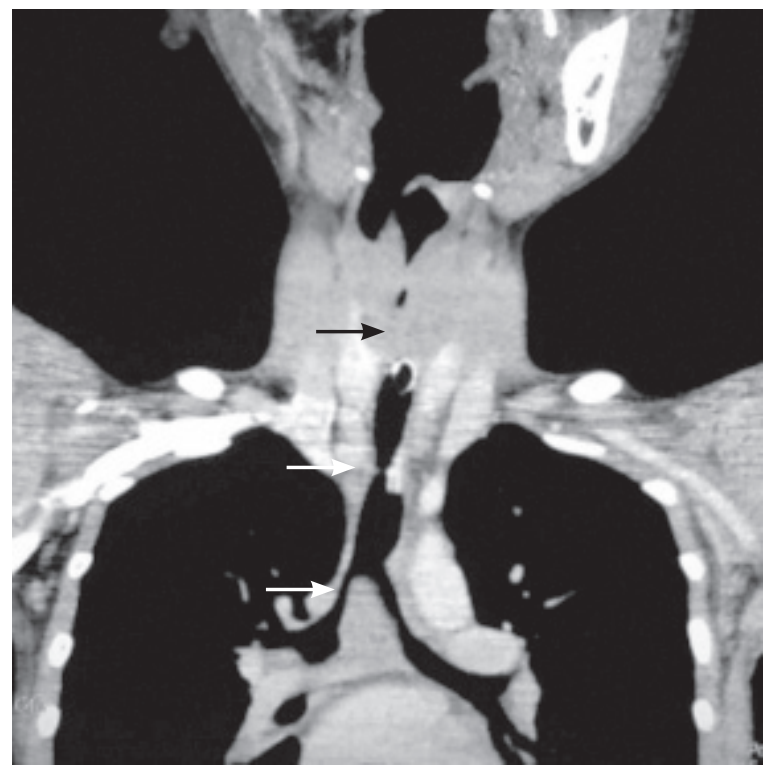

FIgURA 3. Tomografía computada de laringe y tórax, corte sagital. Se observa estenosis traqueal severa (flechas)

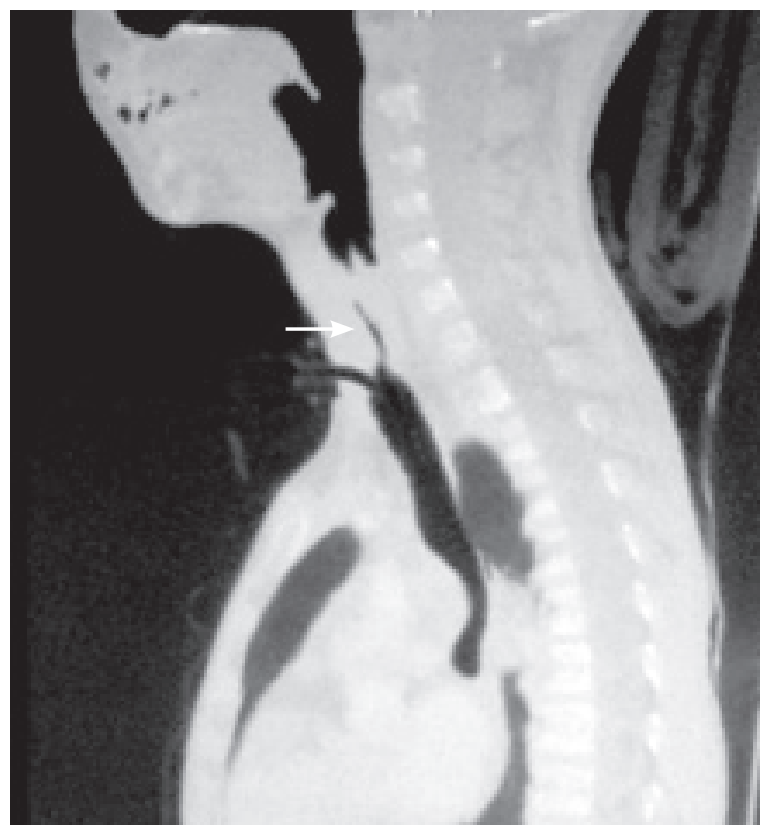


El material de la biopsia traqueal fue insuficiente y el lavado broncoalveolar mostró escasas células escamosas y bronquiales sin atipia. Por no poder descartarse proceso infeccioso subyacente, recibe tratamiento con doxiciclina por posibilidad de rinoscleroma y con trimetropim sulfametoxazol por colonización traqueal con Stenotrophomonas maltophilia.

Durante la hospitalización, se hacen dos dilataciones del ostoma con el objetivo de cambiar la cánula de traqueotomía por una de mayor calibre, acorde con el tamaño del paciente, para asegurar la vía aérea. El paciente se remite a un centro oncológico, en donde se confirma el diagnóstico de leishmaniasis mucosa por muestra de biopsia nasal, que muestra múltiples amastigotes intracelulares destacados con coloración de Giemsa (Figura 4). Se indica tratamiento con antimonio pentavalente por 28 días. A los 6 meses, se realiza una nueva tomografía computada, en la que se observa persistencia de la lesión traqueal con discreta mejoría con respecto a la imagen anterior. El paciente continúa con traqueotomía y en evaluación por Otorrinolaringología para programar la plastía de la vía aérea. No hubo recurrencia de disnea ni de fiebre y se ha recuperado nutricionalmente.

\section{DISCUSIÓN}

La leishmaniasis es un problema de salud pública y es endémica en América Latina. ${ }^{4,8}$ Se estima que la incidencia anual de la forma cutánea es de 1,5 millones y la visceral, de 0,5

FIgURA 4. Histopatología con coloración de Giemsa; múltiples amastigotes intracelulares (flechas)

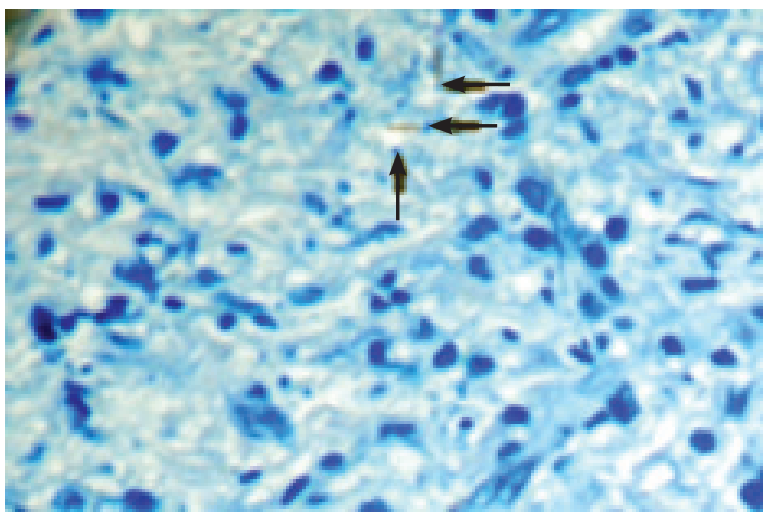

millones, ${ }^{8}$ con una prevalencia global de 12 millones de personas infectadas. En Colombia, el 90,3\% de los casos son de la forma cutánea; $0,4 \%$, mucocutánea; y 0,3\%, visceral..$^{11}$ En Sudamérica, la Leishmania braziliensis es la principal responsable de los casos de leishmaniasis mucosa; en nuestro caso, no se pudo determinar el subtipo. ${ }^{5}$

La mayoría de los casos de leishmaniasis mucosa sin puerta de entrada evidente descritos en la literatura son adultos con factores de riesgo, tales como habitar en área endémica;,1,12,13 inmunosupresión, como diabetes, tabaquismo, consumo de alcohol, trasplante renal; y el uso de corticoides inhalado, este último asociado a leishmaniasis laríngea aislada. ${ }^{1,5,10,14}$

La leishmaniasis laríngea aislada es rara y su fisiopatología aún no es clara. ${ }^{10}$ En la literatura, se menciona un caso de afección traqueal sin estenosis asociado a un compromiso nasal y palatino. ${ }^{13}$ Nuestro caso es el primero descrito de leishmaniasis mucosa con afección traqueal en un niño de 12 años, sin factores de riesgo ambientales o personales, ni condiciones anergizantes concomitantes. El diagnóstico de la localización laríngea es difícil y puede ser confundida con una neoplasia. ${ }^{3,8}$ El compromiso nasal y la obstrucción grave de la vía aérea nos llevaron a pensar en entidades de comportamiento crónico infeccioso, como el rinoescleroma documentado en municipios cercanos a nuestra área de influencia, ${ }^{15}$ tuberculosis, micosis endémicas ${ }^{8} \mathrm{y}$, con menor probabilidad, cáncer ${ }^{8}$ y granulomatosis de Wegener. ${ }^{12}$

Las manifestaciones clínicas de la enfermedad dependen del sitio afectado, de los factores de virulencia propios de cada especie, del tamaño del inóculo, de factores ambientales y de la inmunocompetencia del paciente. ${ }^{8}$ En la leishmaniasis laríngea, es común encontrar disfonía, disfagia, tos, pérdida de peso y fiebre. ${ }^{1,3,13}$ Puede haber lesiones concomitantes de la mucosa nasal y senos paranasales, típicamente no dolorosas, ${ }^{12,13}$ que se asocian a un malestar nasal y rinorrea serosanguinolenta. ${ }^{13}$

Los hallazgos endoscópicos de la leishmaniasis laríngea incluyen ulceración transglótica bilateral, edema perilesional, ${ }^{3}$ hipertrofia de bandas ventriculares, ${ }^{1,14}$ masas exofíticas de apariencia granular ${ }^{14}$ vegetación ulcerada y granulomas en cuerdas vocales., ${ }^{5,14}$ En nuestro paciente, se encontró hipertrofia de la epiglotis y edema glótico y ocupación de la vallécula.

La biopsia es clave en el diagnóstico de la leishmaniasis mucosa. ${ }^{1,12}$ Se describe un caso 
de leishmaniasis con sospecha inicial de granulomatosis de Wegener por el compromiso de senos paranasales, en el que fueron necesarias muchas biopsias para arribar al diagnóstico de certeza. ${ }^{12}$ En nuestro caso, fueron necesarias tres biopsias para el diagnóstico.

La evaluación de la respuesta al tratamiento está basada en criterios clínicos, control semestral de serología y de nasofibrolaringoscopía. La falla terapéutica puede deberse a dosis y tiempo subóptimos. ${ }^{4}$ Aunque la leishmaniasis mucosa responde bien al tratamiento y las cicatrices son raras, ${ }^{10}$ en nuestro caso, debido a la cronicidad $\mathrm{y}$ al retraso en el diagnóstico, las lesiones cicatrizales residuales deformantes de la vía aérea, especialmente la estenosis traqueal, no mejoraron con meglumina antimoniato en dosis y tiempos adecuados.

La leishmaniasis con compromiso aislado de la mucosa laríngea es una entidad rara, especialmente en la población pediátrica. Para su diagnóstico, es indispensable la biopsia del tejido afectado, y su tratamiento oportuno previene secuelas anatómicas permanentes, como la deformidad o la obstrucción de la vía aérea.

\section{BIBLIOGRAFÍA}

1. Fsadni C, Fsadni P, Piscopo T, Mallia-Azzopardi C, et al. Laryngeal leishmaniasis in Malta. J Infect 2007;54(2):e61-3.

2. Prieto Tato LM, La Orden Izquierdo E, Guillén Martín $\mathrm{S}$, Salcedo Lobato E, et al. Diagnóstico y tratamiento de la leishmaniasis visceral infantil. An Pediatr (Barc) 2010;72(5):347-51.

3. Mathur SR, Ray R, Handa KK. Isolated laryngeal leishmaniasis in an immunocompetent patient in India. Trop Doct 2006;36(1):55-6.
4. Amato VS, Tuon FF, Bacha HA, Neto VA, Nicodemo AC. Mucosal leishmaniasis. Current scenario and prospects for treatment. Acta Trop 2008;105(1):1-9.

5. Aliaga L, Cobo F, Mediavilla JD, Bravo J, et al. Localized mucosal leishmaniasis due to Leishmania (Leishmania) infantum: clinical and microbiologic findings in 31 patients. Medicine (Baltimore) 2003;82(3):147-58.

6. Lessa MM, Lessa HA, Castro TW, Oliveira A, et al. Mucosal leishmaniasis: epidemiological and clinical aspects. Braz J Otorhinolaryngol 2007;73(6):843-7.

7. Suárez Rodríguez B, Fernández BI, Santos Sanz S, Sierra Moros MJ, et al. Situación epidemiológica y de los factores de riesgo de transmisión de Leishmania infantum en España. Rev Esp Salud Pública 2012;86(6):555-64.

8. Kopterides P, Mourtzoukou EG, Skopelitis E, Tsavaris $\mathrm{N}$, Falagas ME. Aspects of the association between leishmaniasis and malignant disorders. Trans $R$ Soc Trop Med Hyg 2007;10(12)1:1181-9.

9. Casero R, Laconte L, Fraenza L, Iglesias N, et al. Leishmaniosis laríngea recidivante: un caso inusual en un paciente inmunocompetente tratado con corticoides. Rev Argent Microbiol 2010;42(2):118-21.

10. Faucher B, Pomares C, Fourcade S, Benyamine A, et al. Mucosal Leishmania infantum leishmaniasis: Specific pattern in a multicentre survey and historical cases. J Infect 2011;63(1):76-82.

11. WHO technical report series; no. 949. Control of the leishmaniasis: report of a meeting of the WHO Expert Committee on the Control of Leishmaniasis, Geneva, 22-26 March 2010. [Consulta: 12 de febrero de 2014]. Disponible en: http:/ /www.paho.org/hq/index.php?option=com docman\&task=doc_view\&gid=16979\&Itemid.

12. Brahn E, Pegues DA, Yao Q, Craft N. Mucocutaneous leishmaniasis masquerading as Wegener granulomatosis. J Clin Rheumatol 2010;16(3):125-8.

13. Di Lella F, Vincenti V, Zennaro D, Afeltra A, et al. Mucocutaneous leishmaniasis: report of a case with massive involvement of nasal, pharyngeal and laryngeal mucosa. Int J Oral Maxillofac Surg 2006;35(9):870-2.

14. Bodet E, Andreu L, Ruiz Giner A, Fortuna JC, Palomar V. Leishmaniasis laríngea: presentación de dos casos clínicos. ORL-Dips 2002;29(3):131-4.

15. Marrugo Pardo G, Somogyi Rodríguez M. Escleroma respiratorio en niños: experiencia en el Hospital de la Misericordia. Rev Fac Med (Bogotá) 2002;50(2):65-8. 